\title{
V669 Cep: A new binary system with a $B[e]$ star
}

\author{
A. S. Miroshnichenko ${ }^{1,2}$, K. S. Bjorkman ${ }^{1}$, E. L. Chentsov ${ }^{3,4}$, V. G. Klochkova ${ }^{3,4}$, N. Manset ${ }^{5}$, \\ P. García-Lario ${ }^{6}$, J. V. Perea Calderón ${ }^{7}$, R. J. Rudy ${ }^{8}$, D. K. Lynch ${ }^{8}$, J. C. Wilson ${ }^{9}$, and T. L. Gandet ${ }^{10}$ \\ 1 Ritter Observatory, Dept. of Physics \& Astronomy, University of Toledo, Toledo, OH 43606-3390, USA \\ 2 Central Astronomical Observatory of the Russian Academy of Sciences at Pulkovo, 196140, Saint-Petersburg, \\ Russia \\ 3 Special Astrophysical Observatory of the Russian Academy of Sciences, Karachai-Cirkassian Republic, \\ Nizhnij Arkhyz, 369167, Russia \\ 4 Isaac Newton Institute of Chile, SAO Branch \\ 5 Canada-France-Hawaii Telescope Corporation, 65-1238 Mamalahoa Hwy., Kamuela, HI 96743, USA \\ 6 ISO Data Centre, Science Operations and Data Systems Division of the Research and Scientific Support \\ Department of ESA, Villafranca del Castillo, Apartado de Correos 50727, 28080 Madrid, Spain \\ 7 INSA S.A., Villafranca del Castillo, Apartado de Correos 50727, 28080 Madrid, Spain \\ 8 The Aerospace Corp. M2/266, PO Box 92957, Los Angeles, CA 90009, USA \\ 9 Cornell University, Dept. of Astronomy, 226 Space Sciences Bldg., Ithaca, NY 14853, USA \\ 10 Lizard Hollow Observatory, PO Box 77021, Tucson, AZ 85703-7021 USA
}

Received 1 March 2002 / Accepted 4 April 2002

\begin{abstract}
We present the results of optical and near-IR spectroscopic and broadband multicolour photometric observations of the emission-line object V669 Cep. We find evidence that it contains a hot, low luminosity, B4B6 star and a cool companion (most likely late-type giant). Significant variations of the $\mathrm{H} \alpha$ line strength are detected on a timescale of months. The emission-line spectrum and strong IR-excess indicate a large amount of circumstellar gas and dust in the system. The spectral energy distribution in the near-IR region and the absence of late-type star features in the optical spectrum indicates that the cool star is heavily obscured by circumstellar dust, while the hot star is much less affected by reddening. The system is located at $1-1.5 \mathrm{kpc}$ from the Sun in the local spiral arm. We suggest that V669 Cep is an evolved and probably mass exchanging binary system, a member of the group of Be stars with warm dust.
\end{abstract}

Key words. stars: emission-line, Be - stars: individual: V669 Cep - techniques: spectroscopic, photometric

\section{Introduction}

Over the last few years we have been extensively studying IRAS sources with a steep flux decrease toward longer wavelengths in the region $12-100 \mu \mathrm{m}$, associated with early-type emission-line stars. The presence of a very strong emission in the Balmer lines, many permitted and forbidden Fe II lines, and a significant near-IR excess indicate that they are surrounded by a significant amount of circumstellar gas and dust. This description is similar to that of $\mathrm{B}[\mathrm{e}]$ stars, a group distinguished by Allen \& Swings (1976). However, while the $\mathrm{B}[\mathrm{e}]$ stars include objects of very different nature and evolutionary state, we limit ourselves to a subsample which we tentatively call $B e$ stars with warm dust (Sheikina et al. 2000). They seem to be post-main sequence intermediate- and high-mass stars.

Send offprint requests to: A. S. Miroshnichenko, e-mail: anatoly@physics.utoledo.edu
Some of the objects show evidence of the presence of a cool star in addition to the hot star. These studies resulted in a series of publications (Miroshnichenko et al. 2000, 2001, 2002), where we described the objects' properties in detail for the first time. In this paper we present the results of our observations of V669 Cep, another object which turned out to have similar features to those of our sample.

The object was found in the course of the Tonantzintla Observatory search for emission-line stars (Gonzales \& Gonzales 1956, star No. 71) and then listed by Wackerling (1970) as 3g71. No brightness estimate for the object was reported in these catalogs. 3g71 was detected by two IR satellites, IRAS and MSX (Egan et al. 1999), and listed in their point source catalogs as IRAS $22248+6058$ and MSX5_G106.3912+03.0922, respectively. The noncolour-corrected IRAS fluxes refined using the ADDSCAN 
Table 1. Satellite IR photometry of V669 Cep.

\begin{tabular}{lcccc}
\hline \hline IRAS & $12 \mu \mathrm{m}$ & $25 \mu \mathrm{m}$ & $60 \mu \mathrm{m}$ & $100 \mu \mathrm{m}$ \\
\hline $22248+6058$ & $12.50 \pm 0.04$ & $13.98 \pm 0.04$ & $13.28 \pm 0.13$ & $42.7 \pm 2.3$ \\
\hline MSX & $8.28 \mu \mathrm{m}$ & $12.13 \mu \mathrm{m}$ & $14.65 \mu \mathrm{m}$ & $21.34 \mu \mathrm{m}$ \\
\hline 5C_G106.3912+03.0922 & $8.40 \pm 0.41$ & $9.16 \pm 0.29$ & $9.02 \pm 0.37$ & $10.94 \pm 0.71$ \\
\hline
\end{tabular}

The fluxes are given in Janskys.

procedure (co-addition of all IRAS scans; see Weaver \& Jones 1992) along with those from the MSX survey are listed in Table 1. The IR position coincides with that of the Guide Star Catalog (Lasker et al. 1990) source GSC42640935 with an optical brightness of $12.2 \mathrm{mag}$.

Hang et al. (1999) obtained low-resolution (1.2 $\left.\AA \mathrm{pix}^{-1}\right)$ optical spectra of $3 \mathrm{~g} 71$ in $1993 / 5$ and found a number of weak and moderately strong permitted and forbidden $\mathrm{Fe}$ II lines in emission, $\mathrm{He}$ I lines in absorption, a strong and variable emission in the $\mathrm{H} \alpha$ line, and narrow emission components inside absorption wings of higher lines of the Balmer series $(\mathrm{H} \beta$ and $\mathrm{H} \gamma)$. In 1997/8 Halpern et al. (2001) also obtained a few spectra of the object with a similar resolution $\left(1.5 \AA \mathrm{pix}^{-1}\right)$ in the course of identification of a possible optical counterpart for a $\gamma$-ray source 3EGJ2227+6122. 3g71 has occurred in the positional error circle of 3EGJ2227+6122, but was not identified as its counterpart. The $1997 / 8$ spectra turned out to be very similar to those of $1993 / 5$ and, in addition to the mentioned features, revealed the $\mathrm{H} \delta$ and $\mathrm{H} \epsilon$ lines without obvious emission components.

Hang et al. (1999) mentioned a tentative identification of $3 \mathrm{~g} 71$ with an X-ray source $1 \mathrm{H} 2214+589$ (Tuohy et al. 1988), detected by HEAO-1 with an error box of about $2.5 \times 0.5$ degrees (Wood et al. 1984). Summarizing their spectroscopic data and taking into account the strong IR flux from $3 g 71$ (see Table 1), Hang et al. suggested that the object is more likely a Herbig B5e star rather than a Be/X-ray binary. Even earlier Wood et al. (1984) listed another X-ray source, $4 \mathrm{U} 2238+60$ which is located almost at the border of the HEAO- 1 error box for $1 \mathrm{H} 2214+589$, as a possible counterpart of the latter. This was supported by in't Zand et al. (2000), who discovered that the position of the weak transient X-ray source SAXJ2239.2+6116 coincided with that of $4 \mathrm{U} 2238+60$. The transient was found to show regular outbursts with a period of 232 days and have an optical counterpart, a 16-mag Be star. Thus, the association of $3 \mathrm{~g} 71$ and $1 \mathrm{H} 2214+589$ was not confirmed.

Recently Kazarovets, Samus, \& Durlevich (2001) included the object in the General Catalog of Variable Stars referring to Takamizawa ${ }^{1}$, who obtained a few photovisual observations of $3 g 71$ in 1994-1999 showing brightness variations from 11.9 to $12.4 \mathrm{mag}$ (in a nonstandard passband close to the $V$-band). The star was denoted as V669 Cep with the variability type SR: (possible semi-regular variable).

\footnotetext{
1 The light curve is accessible from http://www.kusastro. kyoto-u.ac.jp/vsnet/LCs/index/CEPV669.html
}

No other ground-based photometric data have been published so far. All the information about V669 Cep = $3 \mathrm{~g} 71$ mentioned above is not sufficient to make any definite conclusion about the nature and evolutionary state of this object. To clarify this issue we obtained new observations of V669 Cep and discuss them here.

\section{Observations}

The photometric $W B V R$ observations of V669 Cep were obtained on 2001 December 16 at the Tien-Shan Observatory (TSAO, Kazakhstan) using a 50-cm telescope with a standard pulse-counting single-channel photometer. The instrumental system, whose $B V R$ bands are very close to the Johnson system, is described in Khaliullin et al. (1985). The errors of individual measurements were $0.02 \mathrm{mag}$ in all the bands. HD 213530 (B9), whose $W B V R$ magnitudes are listed in Kornilov et al. (1991), was used as a comparison star. The instrumental system stability was controlled using other standard stars observed during the night. The Johnson $U-B$ colour-index of HD 213530 was calculated from the $W-B$ using a linear regression, derived for nearly 1000 B7-A9 non-supergiant stars from the Bright Star catalogue with $W B V R$ photometry $(U-B=(0.830 \pm 0.004)(W-B)-0.006 \pm 0.001)$. Its Johnson $R-I$ colour-index was calculated from the reddening $\left(E_{B-V}=0.12 \mathrm{mag}\right)$ and a calibration of Wegner (1994).

Differential $B V R I$ CCD photometry of V669 Cep was obtained with the 28-cm Beardsley Schmidt-Cassegrain telescope at the Lizard Hollow Observatory (LHO, Tucson, AZ, USA) on five nights, from December 2001 through January 2002. An SBIG ST-7E camera was used, and the frames were measured with the SBIG CCDOPS software. First-order extinction coefficients were determined for each night's observations. Standard stars, taken from Kornilov et al. (1991) and other literature sources, were observed from which transformation coefficients between the instrumental and Johnson system were derived. The primary comparison and check stars were, respectively, HD 213530 and HD 212776. Neither of those stars was used in determining the transformation coefficients. Frames of the primary comparison stars were not obtained each night. The magnitudes and colours of V669 Cep were measured with respect to HD 213530. V669 Cep did not show any brightness variations exceeding the internal observational errors, which were $0.03-0.1 \mathrm{mag}$ in the $B$-band 
Table 2. Optical and near-IR photometry of V669 Cep.

\begin{tabular}{rccccccrrr}
\hline \hline JD2452000+ & $V$ & $U-B$ & $B-V$ & $V-R$ & $V-I$ & $J$ & $H$ & $K$ & Remark \\
\hline 260.109 & 12.47 & 0.00 & 0.74 & 0.85 & & & & TSAO $^{\mathrm{a}}$ \\
$246-283$ & 12.45 & - & 0.73 & 0.90 & 1.50 & & & LHO $^{\mathrm{b}}$ \\
118.65 & & & & & & 9.98 & 9.04 & 7.61 & CST $^{2}$ \\
220.50 & & & & & & 10.0 & 9.1 & 7.7 & Palomar \\
\hline & 6.98 & -0.20 & 0.03 & 0.07 & 0.09 & & & HD 213530 \\
\hline
\end{tabular}

${ }^{a} U-B$ was calculated from $W-B=-0.05 \mathrm{mag}$ using the same procedure as described for HD 213530.

b The data were obtained between JD 2452246 and JD 2452283; the star is too weak in the $U$-band frames.

The near-IR photometric errors are $0.05 \mathrm{mag}$ for the $H K$ CST data, otherwise 0.1 mag.

and 0.01-0.03 mag in the VRI-bands. All our optical photometric data are presented in Table 2.

On 2001 July 27 we obtained $J H K$ photometry of V669 Cep at the 1.55-m Carlos Sánchez Telescope (CST), operated by the Instituto de Astrofísica de Canarias at the Spanish Observatorio del Teide (Tenerife, Spain). We used a CVF infrared spectrophotometer equipped with an InSb photovoltaic detector, operating at the temperature of liquid nitrogen, with a photometric aperture of $15^{\prime \prime}$ and a chopper throw of $30^{\prime \prime}$ in the E-W direction to subtract the contribution from the background sky. The Teide photometric system is described in Arribas \& Martínez-Roger (1987), as well as its relations with other standard photometric systems.

The infrared low-resolution spectroscopic observations of V669 Cep were acquired on 2001 July 29 at the 60-inch telescope of the Palomar Observatory with the CORMASS spectrograph (Wilson et al. 2001) in the region 0.75$2.5 \mu \mathrm{m}(R \sim 300)$. The instrumental response and most of the effects of atmospheric absorption were removed by dividing the spectrum of V669 Cep by that of the calibrator star (HD 21338, G8 v, $V=7.45 \mathrm{mag}$ ). To remove the intrinsic spectrum of the calibrator from this ratio, a model from Kurucz (1994) appropriate to a G8 v star was used. The resulting spectrum was converted into magnitudes using passbands of the $J H K$ photometric system from Bessell \& Brett (1988). These magnitudes are presented in Table 2 along with the CST data. Both sets of magnitudes agree with each other rather well, justifying the reliability of the near-IR spectrum calibration. Additionally, from these data we estimated the Johnson I-band magnitude (10.9 mag) using a calibration by Straizys (1977). This turned out to be very close to our LHO data.

The high-resolution optical spectra of V669 Cep were obtained with the following telescopes and spectrometers:

1. 6-m telescope of the Special Astrophysical Observatory (SAO) of the Russian Academy of Sciences: on 2001 January 2 with the prime-focus échelle-spectrometer PFES (Panchuk et al. 1998) and a $1140 \times 1170$ pixel CCD detector in a spectral range 5000-6650 $\AA$ with a mean $R \sim 15000$; and on 2001 November 29 with the échelle-spectrometer Lynx (Panchuk et al. 1999), mounted in the Nasmyth-2 focus, and the same CCD detector in a range 5030-6680 $\AA$ with a mean $R \sim$ 30000 . The SAO spectra were reduced using standard methods under MIDAS;

2. on 2001 September 7 at the 3.6-m CFHT (Mauna Kea, Hawaii, USA) with the Coudé échelle spectrometer Gecko and a $2048 \times 4608$ thinned back-illuminated EEV chip in the range $6505-6610 \AA$ and with a $R \sim$ 60000 ;

3. on 2001 October 10 at the 2.1-m Otto Struve telescope of the McDonald Observatory (Mt. Locke, Texas, USA) with the Sandiford échelle-spectrometer (McCarthy et al. 1993) in the range 5540-6915 $\AA$ and with a $R \sim 60000$. A $1200 \times 400$ pixel CCD was used.

The CFHT and McDonald spectra were reduced with IRAF $^{2}$.

\section{Results}

\subsection{Spectrum}

Both our high-resolution optical spectra and lowresolution near-IR spectrum of V669 Cep contain emission lines of neutral hydrogen, permitted and forbidden Fe II and $\mathrm{O}$ I lines. The emission-line spectrum is variable. Some iron lines at $\lambda \lambda 5150-5400 \AA$ are seen only in the January 2001 spectrum, while others only in the November 2001 spectrum (Fig. 1a). This may be due in part to a low signal-to-noise ratio ( $\sim 60$ and $\sim 40$ in January and November, respectively), since all of these lines are weak. However, the variations are clearly seen in the [O I] lines and $\mathrm{H} \alpha$.

The very weak emission lines of $[\mathrm{N} \mathrm{II}]$ at 6548 and $6583 \AA$ appeared in our high-resolution spectra. We also detected three He I lines with different profile shapes. The He I $6678 \AA$ line has a pure absorption profile, the $5876 \AA$ line exhibits a weak and variable emission component (Fig. 1b), while the $10830 \AA$ line (observed at low resolution) is completely in emission. Other absorption features, seen in the spectra, are weak diffuse interstellar

\footnotetext{
${ }^{2}$ IRAF is distributed by the National Optical Astronomy Observatories, which are operated by the Association of Universities for Research in Astronomy, Inc., under contract with the National Science Foundation.
} 

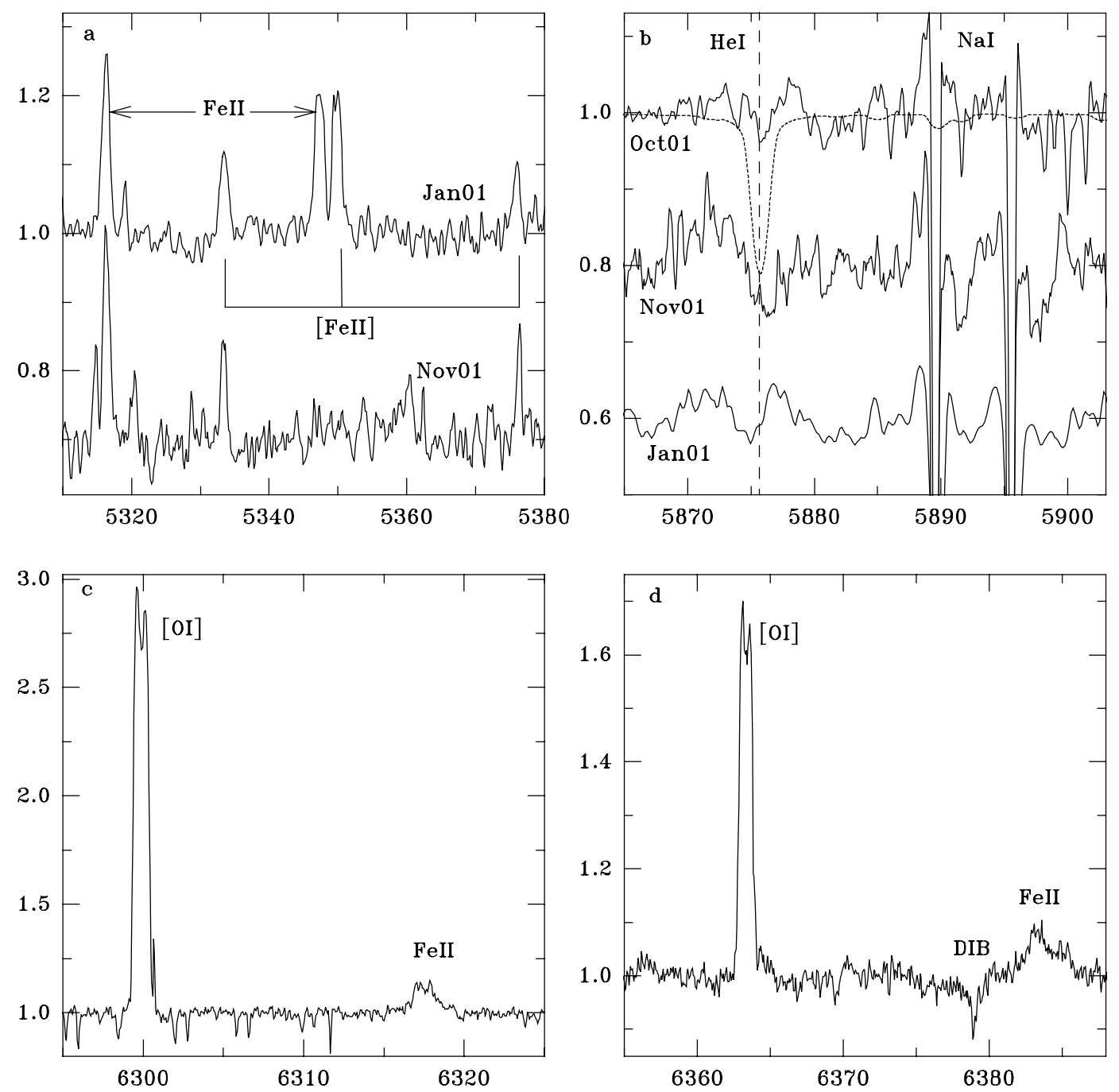

Fig. 1. Portions of the spectrum of V669 Cep. a) The SAO January and November 2001 spectra shifted by $0.2 I_{\mathrm{c}}$ with respect to each other. b) The He I and Na I lines region. The vertical line represents the laboratory wavelength of the $5876 \AA$ line. A synthetic spectrum for $T_{\text {eff }}=15000 \mathrm{~K}, \log g=4.0$, and a rotation velocity of $50 \mathrm{~km} \mathrm{~s}^{-1}$ is shown by the dotted line. c) and d) Portions of the McDonald spectrum in the regions of [O I] lines. Heliocentric wavelengths are given in $\AA$, while the intensities are normalized to the continuum.

bands (DIBs), and a group of features at $2.29-2.39 \mu \mathrm{m}$. The latter can be identified with the $\mathrm{CO}(2-0),(3-1)$, $(4-2)$, and (5-3) bands. The only Fe II lines, seen in the near-IR spectrum, are those at 9997 and $10501 \AA$ (Rudy et al. 2000 and references therein). These lines, although much stronger, are also observed in the spectra of luminous B[e] stars (Lopes et al. 1992).

The list of lines identified in the spectrum of V669 Cep with the help of a catalogue by Coluzzi (1993) is presented in Tables 3 (optical range) and 4 (near-IR range). Parts of the spectra are shown in Figs. 1-4.

Overall, our optical spectra are similar to the lowresolution spectra of Hang et al. (1999). The only Balmer line from our spectral range, $\mathrm{H} \alpha$, is extremely strong and rather narrow (full width at half maximium, FWHM $120 \mathrm{~km} \mathrm{~s}^{-1}$; Fig. 2). The intensity of its central peak varies dramatically with time (Fig. 2a). The signal registered in the underlying continuum was high $(\geq 1000$ counts $)$ in all our spectra, while the line's peak was not saturated. This excludes significant normalization errors. The existing $\mathrm{H} \alpha$ $E W$ data are presented in Table 5. Due to a higher resolution and signal-to-noise ratio, we detected the absorption wings of the $\mathrm{H} \alpha$ line in the CFHT spectrum. Their slope is consistent with an effective temperature $\left(T_{\text {eff }}\right)$ of $\sim 15000 \mathrm{~K}$ and a $\log g \geq 3$.

The DIBs are weak, indicating a low reddening. For example, the equivalent width of the strongest DIB at $5780 \AA(0.23 \AA)$ corresponds to $E_{B-V} \sim 0.5 \mathrm{mag}$ (Herbig 1993). Rudy et al. (1991) have used the IR O I lines to estimate the reddening when the 8446 and $11287 \AA$ lines are produced mainly by $\mathrm{Ly}_{\beta}$ fluorescence, and continuum fluorescence can be treated as a perturbation. In V669 Cep, however, it is clear from the observed ratios that continuum fluorescence makes a large contribution. The difficulty is that for continuum fluorescence the relative strengths of the three lines depend on the UV spectral 
Table 3. Lines identified in the optical spectrum of V669 Cep.

\begin{tabular}{|c|c|c|c|c|c|c|c|c|c|c|c|}
\hline Line ID & $\lambda_{\text {lab }}$ & $I / I_{\mathrm{c}}$ & $\begin{array}{l}R V \\
\mathrm{~km} \mathrm{~s}^{-1}\end{array}$ & $\begin{array}{l}E W \\
\AA\end{array}$ & Rem. & Line ID & $\stackrel{\lambda_{\text {lab }}}{\AA}$ & $I / I_{\mathrm{c}}$ & $\begin{array}{l}R V \\
\mathrm{~km} \mathrm{~s}^{-1}\end{array}$ & $\begin{array}{l}E W \\
\AA\end{array}$ & Rem. \\
\hline 1 & 2 & 3 & 4 & 5 & 6 & 1 & 2 & 3 & 4 & 5 & 6 \\
\hline Fe II(42) & 5018.44 & 1.3 & -34 & 0.54 & Jan01 & He I(11) & 5875.72 & 0.95 & - & & \\
\hline$[\mathrm{Fe} \mathrm{II}](19 \mathrm{~F})$ & 5158.78 & 1.2 & $-24:$ & & Nov01 & $\mathrm{Na} \mathrm{I}(1)$ & 5889.95 & 0.49 & -16 & 0.41 & \\
\hline$[\mathrm{Fe} I \mathrm{II}](35 \mathrm{~F})$ & 5163.95 & 1.1 & $-17:$ & & Nov01 & $\mathrm{Na} I(1)$ & 5895.92 & 0.57 & -16 & 0.43 & \\
\hline Fe II $(42)$ & 5169.03 & 1.3 & -21 & 0.26 & & Fe II $(74)$ & 6147.74 & 1.02 & - & 0.02 & \\
\hline$[\mathrm{Fe} \mathrm{II}](19 \mathrm{~F})$ & 5220.06 & 1.1 & $-24:$ & & Nov01 & DIB & 6195.96 & 0.94 & -13 & 0.08 & \\
\hline Fe II $(49)$ & 5234.62 & 1.1 & -22 & 0.08 & Jan01 & DIB & 6203.08 & 0.94 & -14 & 0.04 & \\
\hline Fe II(41) & 5238.58 & 1.1 & -20 & $0.15:$ & Jan01 & $\mathrm{Fe} \mathrm{II}(74)$ & 6247.55 & 1.08 & - & $0.07:$ & $+\mathrm{f}$ \\
\hline Fe II(41) & 5256.89 & 1.1 & $-30:$ & 0.10 : & Jan01 & Fe II ( ) & 6248.9 & 1.08 & - & & \\
\hline$[\mathrm{Fe}$ II] $](19 \mathrm{~F})$ & 5261.61 & 1.2 & -16 & 0.05 & & {$[\mathrm{O} \mathrm{I}](1 \mathrm{~F})$} & 6300.3 & $2.9-4.0$ & -18 & $2.0-3.1$ & \\
\hline$[\mathrm{Fe} \mathrm{II}](18 \mathrm{~F})$ & 5273.38 & 1.1 & $-21:$ & 0.14 & & $\mathrm{Fe}$ II & 6317.99 & 1.1 & -25 : & 0.3 & \\
\hline Fe II(49) & 5276.00 & 1.1 & $-26:$ & & Nov01 & {$[\mathrm{O} \mathrm{I}](1 \mathrm{~F})$} & 6363.78 & $1.7-1.9$ & -18 & $0.7-1.0$ & \\
\hline Fe II $(49,48)$ & 5316.61 & 1.3 & -22 & 0.34 & & Fe II & 6383.72 & 1.1 & - & 0.24 & \\
\hline$[\mathrm{Fe} I \mathrm{II}](19 \mathrm{~F})$ & 5333.65 & 1.1 & -16 & 0.18 & & {$[\mathrm{~N} \mathrm{II}](1 \mathrm{~F})$} & 6548.03 & 1.04 & -25 & 0.04 & CFHT \\
\hline $\mathrm{Fe}$ II $(49)$ & 5346.56 & 1.2 & -23 & 0.27 & $+\mathrm{f}$ & $\mathrm{H} \alpha$ & 6562.81 & var. & -25 & & \\
\hline$[\mathrm{Fe}$ II $](18 \mathrm{~F})$ & 5347.67 & - & - & - & Jan01 & {$[\mathrm{N} \mathrm{II}](1 \mathrm{~F})$} & 6583.45 & 1.1 & -23 & 0.07 & CFHT \\
\hline$[\mathrm{Fe}$ II $](19 \mathrm{~F})$ & 5376.47 & 1.1 & $-28:$ & 0.09 & & DIB & 6613.56 & 0.85 & -15 & 0.15 & \\
\hline DIB & 5780.37 & 0.85 & -10 & 0.23 & & He I(46) & 6678.15 & 0.95 & - & & Oct01 \\
\hline DIB & 5796.96 & 0.91 & -10 & 0.08 & & & & & & & \\
\hline
\end{tabular}

Comments to the line list in the optical spectrum of V669 Cep.

Unreliable identifications and less accurate measurements are denoted by colons. Intensities at line peaks in units of the underlying continuum are given in Col. 3, heliocentric $R V$ in Col. 4, equivalent widths in Col. 5, and Remarks in Col. 6.

"f" referes to the following line; lines detected in a particular spectrum are referred by dates; Intensities, $R V \mathrm{~s}$ and $E W \mathrm{~s}$ are averaged if detected in more than one spectrum; the $\mathrm{H} \alpha$ equivalent widths are given in Table 5 .

Table 4. Lines identified in the near-IR spectrum of V669 Cep.

\begin{tabular}{llllll}
\hline \hline$\lambda_{\text {lab }}$ & ID & $I / I_{\mathrm{c}}$ & $\lambda_{\text {lab }}$ & ID & $I / I_{\mathrm{c}}$ \\
\hline 8446.35 & O I $(4)$ & 1.24 & 13164.00 & O I & 1.12 \\
9229.017 & P9 & 1.18 & 15556.45 & Br16 & 1.03 \\
9545.974 & P8 & 1.10 & 15700.66 & Br15 & 1.05 \\
9997.58 & Fe II & 1.07 & 15880.54 & Br14 & 1.04 \\
10049.38 & P7 & 1.21 & 16109.31 & Br13 & 1.07 \\
10501.00 & Fe II & 1.05 & 16407.19 & Br12 & 1.09 \\
10830.34 & He I $(1)$ & 1.16 & 16806.52 & Br11 & 1.10 \\
10938.09 & P6 & 1.44 & 17362.11 & Br10 & 1.09 \\
11287.00 & O I & 1.13 & 21655.29 & Br $\gamma$ & 1.16 \\
12818.05 & P5 & 1.95 & & & \\
\hline
\end{tabular}

energy distribution of the exciting source (Grandi 1975, 1976). The reddening can be estimated, but the uncertainty is greater than the case where $\mathrm{Ly} \beta$ fluorescence is the primary source and the intrinsic $8446 / 11287$ intensity ratio is well known. The relative photon flux ratios for continuum illumination by a B-type star are approximately $1 / 0.06 / 0.5$ for the lines at 8446,11287 , and $13164 \AA$, respectively. Using this and allowing for a contribution from $\mathrm{Ly}_{\beta}$ fluorescence gives $E_{B-V}=1.2 \pm 0.3 \mathrm{mag}$. For this we have corrected the strength of the $11287 \AA$ line for telluric absorption assuming a $F W H M$ for the line of $90 \mathrm{~km} \mathrm{~s}^{-1}$ and a heliocentric radial velocity $(R V)$ of $-22 \mathrm{~km} \mathrm{~s}^{-1}$ (values derived from our high-resolution optical measure-
Table 5. The exisiting $\mathrm{H} \alpha$ line equivalent width data for V669 Cep.

\begin{tabular}{crc}
\hline \hline JD2400000+ & $E W, \AA$ & Ref. \\
\hline 49297 & 103 & 1 \\
50006 & 75 & 1 \\
50720 & 88 & 2 \\
51001 & 93 & 2 \\
51912 & 181 & 3 \\
52160 & 67 & 3 \\
52192 & 123 & 3 \\
52243 & 187 & 3 \\
\hline
\end{tabular}

References: 1 - Hang et al. (1999), 2 - Halpern et al. (2001), 3 - this work

ments) in the manner described in the appendix of Rudy et al. (1991).

The emission lines of [O I] at 6300 and $6363 \AA$ have $R V \mathrm{~s}$ close to those of other emission lines and are formed in the object's envelope. In the McDonald spectrum $(R=$ 60000 ) they appear double-peaked (as well as the Fe II lines at 6317 and $6383 \AA$ ) with a mean peak separation of $25 \mathrm{kms}^{-1}$.

The $\mathrm{Na}$ I $\mathrm{D}_{1,2}$ lines consist of a rather deep interstellar absorption component (not split even at $R=60000$ ) superposed on a weak, circumstellar emission (see Fig. 1b). Another absorption component at $+90 \mathrm{~km} \mathrm{~s}^{-1}$ is clearly seen in both D-lines only in the November 2001 spectrum. 

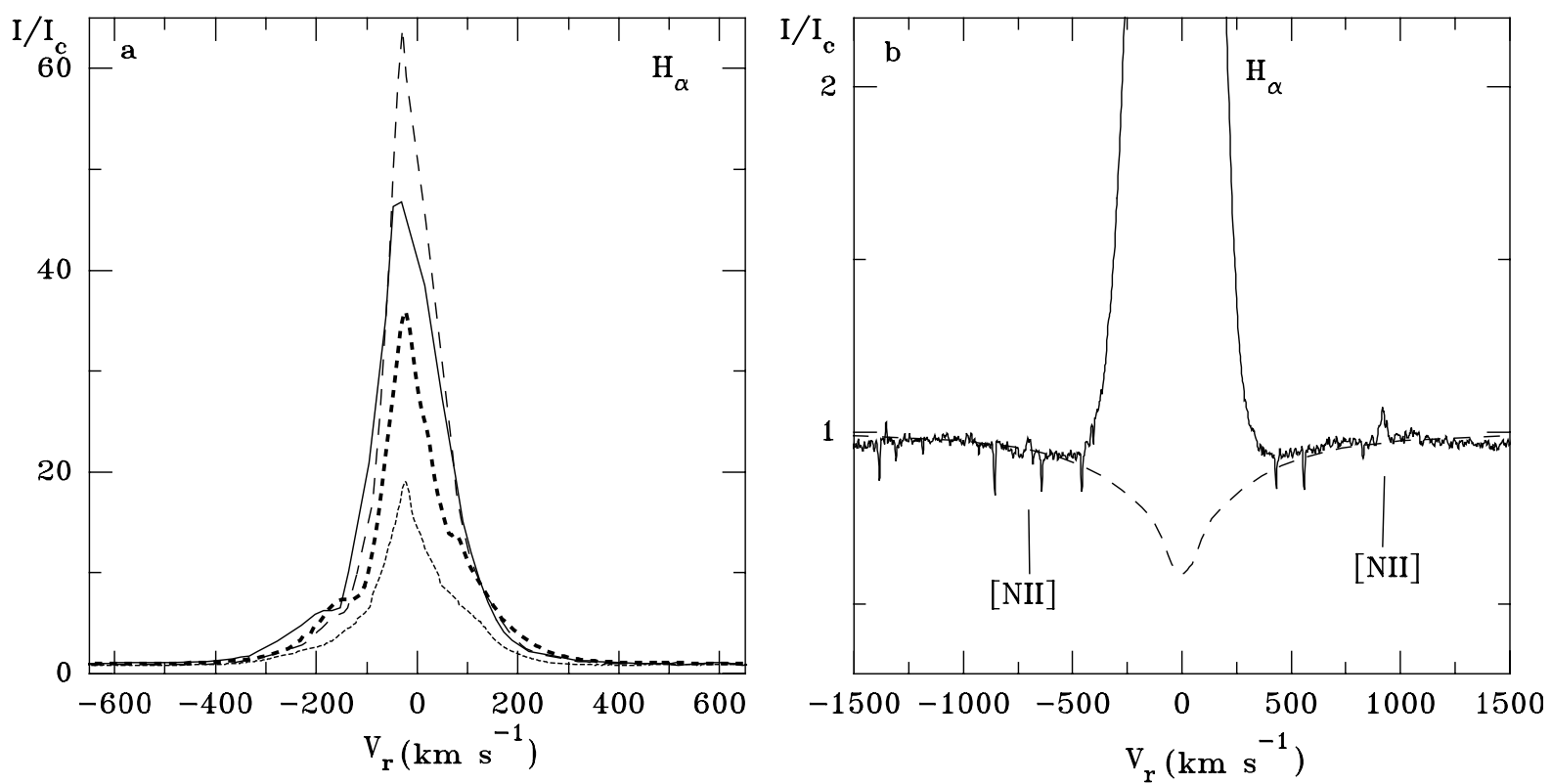

Fig. 2. The H $\alpha$ line profiles of V669 Cep. a) The SAO January 2001 (solid line), CFHT (short-dashed line), McDonald (dotted line), and SAO November 2001 (long-dashed line) spectra. b) The lower part of the CFHT profile (solid line) with a theoretical profile for $T_{\text {eff }}=15000 \mathrm{~K}, \log g=4.0$ and a rotation velocity of $50 \mathrm{~km} \mathrm{~s}^{-1}$ (dashed line). The $R V \mathrm{~s}$ are heliocentric, while the intensity scale is the same as in Fig. 1. Telluric lines were not removed from the spectrum.
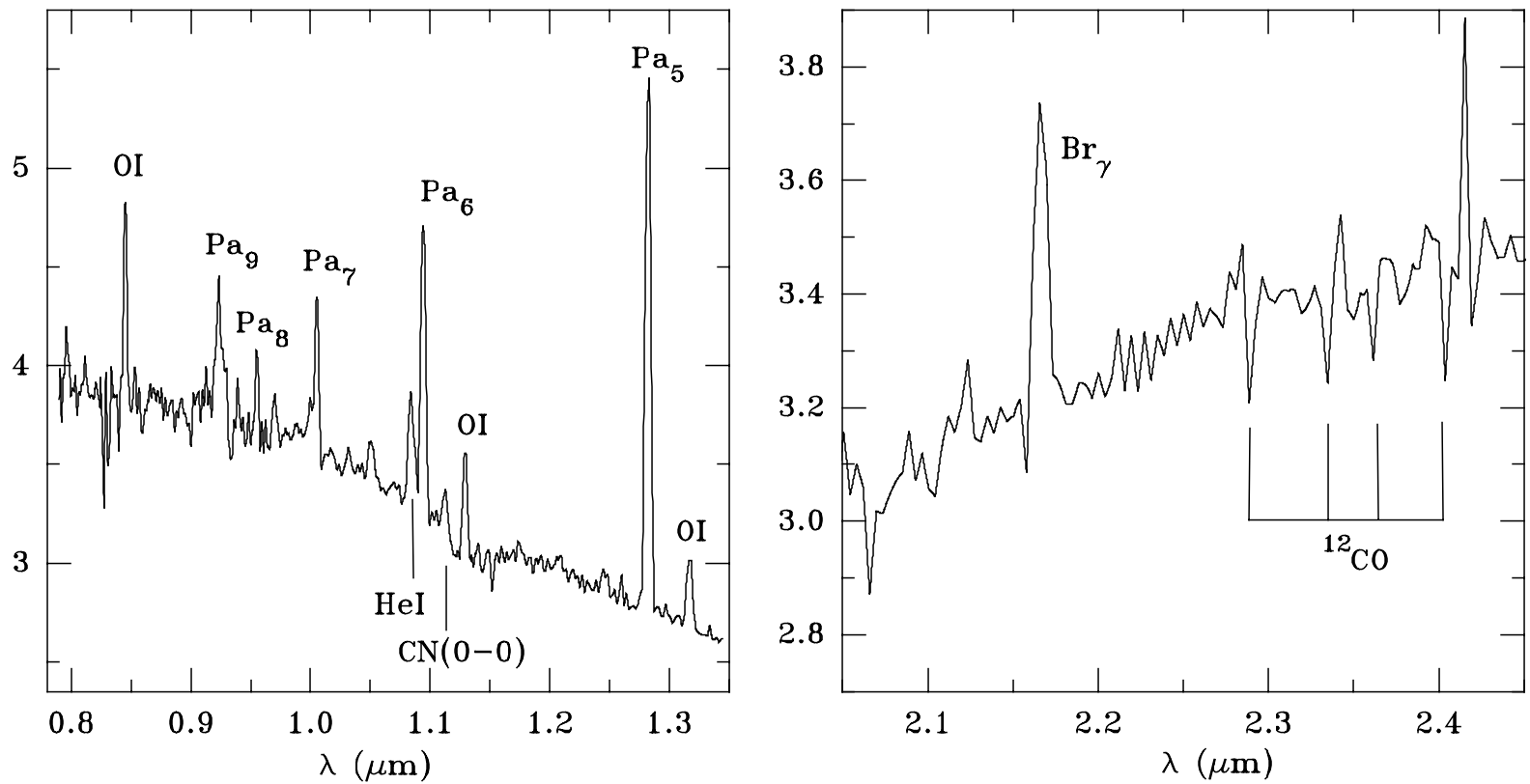

Fig. 3. Parts of the low-resolution near-IR spectrum of V669 Cep. Wavelengths are given in microns, while the fluxes are given in units of $10^{-17} \mathrm{~W} \mathrm{~cm}^{-2} \mu \mathrm{m}^{-1}$.

Unfortunately, it is affected by a telluric line (seen in the McDonald spectrum, the upper plot in Fig. 1b). However if real, it is definitely circumstellar and variable. In the November 2001 spectrum the appearance of this component is accompanied by a positive velocity shift of the He I $5876 \AA$ line. The explanation of these phenomena is not clear yet and requires follow-up high-resolution spectroscopy.

The mean $R V$ of the Na I D interstellar components is $-16 \mathrm{~km} \mathrm{~s}^{-1}$, that of the DIBs is $-12 \pm 3 \mathrm{~km} \mathrm{~s}^{-1}$, while that of the emission lines is $-22 \pm 3 \mathrm{~km} \mathrm{~s}^{-1}$ and $-20 \pm 2 \mathrm{~km} \mathrm{~s}^{-1}$ (January and November 2001, respectively). Both the structure of the interstellar $\mathrm{Na}$ I components and the $R V$ values suggest that the object belongs to the local spiral arm, whose outer edge is located at a distance $(D)$ of $\sim 1-1.5 \mathrm{kpc}$ from the Sun (Efremov 1989). Additional absorption components, formed in the more distant Perseus arm, appear in the spectra of stars in this direction at $R V \mathrm{~s} \sim-45 \mathrm{kms}^{-1}$ and $D \geq 2 \mathrm{kpc}$ (e.g., Munch 1957). This sets an upper limit for $D$ toward V669 Cep at $1.5 \mathrm{kpc}$, 


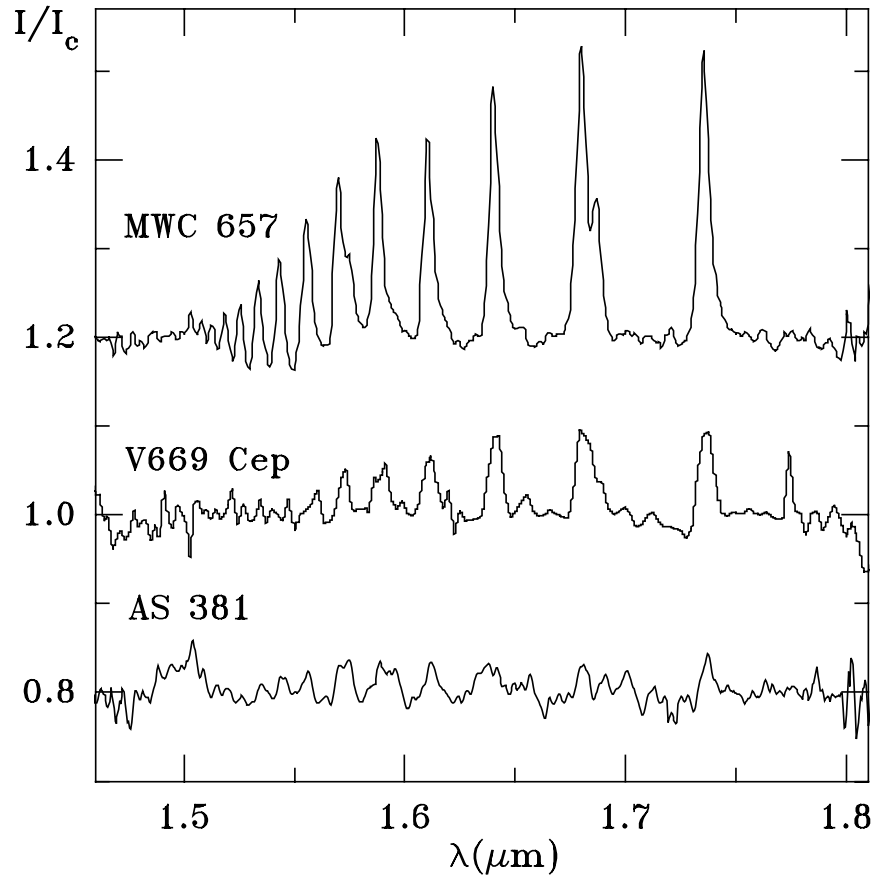

Fig. 4. The normalized $H$-band spectra of several Be stars with warm dust. The spectra of MWC 657 and AS 381, obtained at Lick in July of 2000 with a similar resolution (see Miroshnichenko et al. 2002 for details), are shown for comparison.

considering that the object is unlikely to reside in the inter-arm space.

There is also a continuum jump at $1.1 \mu \mathrm{m}$ (see Fig. 3), which appears in the spectra of stars later than G5 of luminosity types I-III (Wallace et al. 2000) and is attributed to the CN 0-0 band. Along with the presence of the CO absorption bands, this suggests the presence of a late-type component in the system. The absence of late-type star features shortward of $6800 \AA$ does not allow us to constrain its spectral type, and indicates that the cool star is significantly fainter than the hot one.

\subsection{Photometry and spectral energy distribution}

The optical photometry indicates that V669 Cep is a reddened B-type star. From the existing $U B V$ data for stars in a $1^{\circ} \times 1^{\circ}$ region around the object a colour-excess ratio $E_{U-B} / E_{B-V}=0.60$ can be derived. Its application to the colour-indices of V669 Cep gives a spectral type of B5 and $E_{B-V}=0.9 \mathrm{mag}$ (Wegner 1994). The colourexcess is consistent with the estimate from the IR line strengths and larger than that derived from the DIBs strength. The latter is less reliable as showing significantly different relationships in different directions (see Herbig 1993). The nearby stars reddening also indicates that $E_{B-V} \geq 0.7 \mathrm{mag}$ corresponds to $D \geq 1 \mathrm{kpc}$, which is in agreement with our estimate from the $\mathrm{Na}$ I lines and can be adopted as a lower limit for the distance toward V669 Cep.

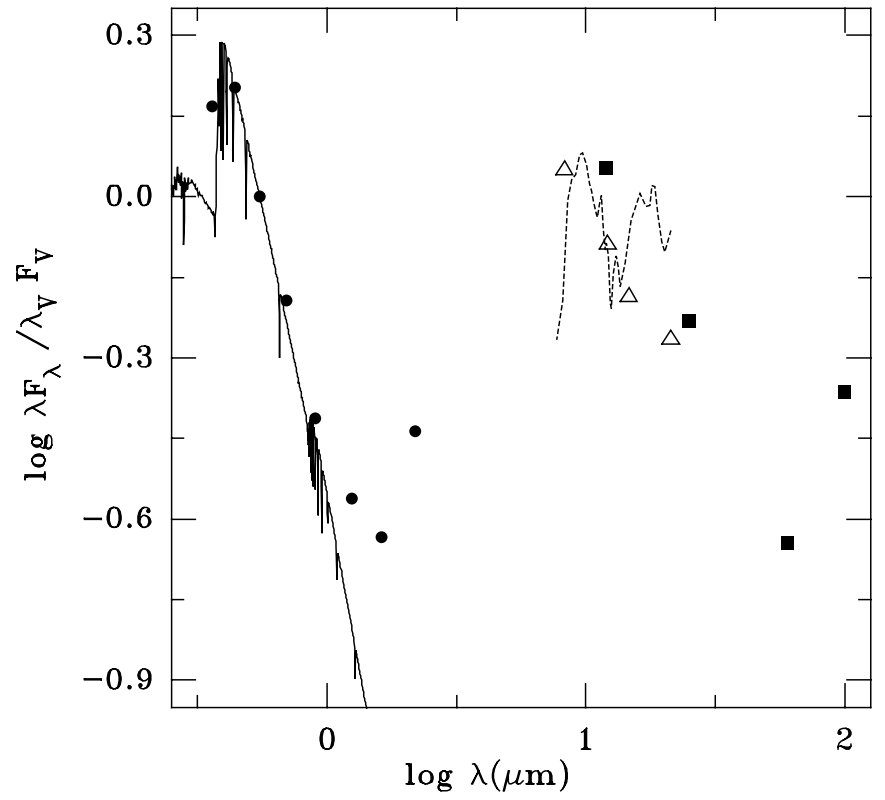

Fig. 5. The SED of V669 Cep. The solid line represents the synthetic spectrum for $T_{\text {eff }}=15000 \mathrm{~K}$ and $\log g=4.0$ from Kurucz (1994). The $U B V R I J H K$ fluxes are shown by filled circles, the IRAS data by filled squares, the MSX data by open triangles, and the IRAS LRS spectrum (rescaled to the $12 \mu \mathrm{m}$ flux) by the dashed line. A reddening of $E_{B-V}=0.9$ mag and $V=12.4$ mag were assumed.

Our near-IR photometry and spectrum show the presence of circumstellar dust, as the colour-indices $(\overline{J-H}=$ $0.94 \pm 0.11, \overline{H-K}=1.43 \pm 0.07)$ are much larger than those expected from a stellar photosphere and the low reddening estimated above. Moreover, the IRAS and MSX data show even stronger mid- and far-IR excesses. The IRAS LRS spectrum, which is rather noisy due to the closeness of the object's flux to the threshold of the spectrometer, was cleaned with a numerical high-frequency filter. This procedure revealed the presence of the silcate emission features at 9.7 and $18 \mu \mathrm{m}$, indicating that the circumstellar dust is oxygen-rich and is optically thin at these wavelengths. The $100 \mu \mathrm{m}$ IRAS flux is most likely contaminated by the interstellar IR cirrus emission, because the $2 \mathrm{D}$ coadded image of the source is extended, and the parameter CIR3 $=114 \mathrm{MJy} \mathrm{sr}^{-1}$ (the total $100 \mu \mathrm{m}$ sky surface brightness around the object) is much larger than the object's $60 \mu \mathrm{m}$ flux (Ivezić \& Elitzur 1995). Hence the $100 \mu \mathrm{m}$ IRAS flux of V669 Cep is not reliable.

The dereddened spectral energy distribution (SED) of V669 Cep (see Fig. 5) shows that an excess radiation above the photospheric spectrum already exists at $\lambda \sim 1 \mu \mathrm{m}$ and rises drastically longward of $\lambda \sim 1.5 \mu \mathrm{m}$. The excess maximum, located at $\sim 10 \mu \mathrm{m}$, suggests that the bulk of circumstellar dust has temperatures $\sim 300 \mathrm{~K}$. The steep flux decrease longward of $20 \mu \mathrm{m}$ and the spectral features discussed above make V669 Cep similar to objects from the group of Be stars with warm dust, recently defined by us (Sheikina et al. 2000). 
The $12 \mu \mathrm{m}$ IRAS flux is 1.5 times higher than the corresponding MSX flux. In part this may be due to the broader IRAS bandpass. The colour-correction factor for this wavelength (1.41), suggested in The IRAS Explanatory Supplement (1985) and based on an A0-type photospheric underlying spectrum, would remove this difference. However, the SED of V669 Cep in the mid-IR is certainly much flatter than that of an A0-type star implying a smaller flux reduction. Nevertheless, the IRAS and MSX fluxes of V669 Cep, obtained 13 years apart, are close to each other. This fact along with the high positional accuracy of the MSX mission provide evidence that the IR excess does belong to V669 Cep and the near-IR flux rise with wavelength is real.

\section{Discussion}

The results of our observations suggest that V669 Cep is most likely a binary system with a hot and a cool stellar component and gas-and-dust circumstellar envelope. Its emission-line spectrum and SED in the IR region are similar to those of other binary Be stars with warm dust, MWC 623 (Zickgraf 2001) and AS 381 (Miroshnichenko et al. 2002). Let us consider different hypotheses about the nature and evolutionary state of the V669 Cep system.

From the object's mean brightness and our estimates for $D$ and $A_{V}$ one can derive an absolute visual magnitude of the hot companion, $M_{V}=-0.9 \pm 0.4 \mathrm{mag}$, assuming no contribution of the cool companion to the $V$-band brightness. The latter suggestion is justified by the absence of noticeable late-type star features in the observed parts of the optical spectrum. According to a calibration of Straizys \& Kuriliene (1981), such an $M_{V}$ corresponds to main-sequence B4-B6 stars. The same range of spectral types follows from our $U B V$ data. Thus, we confirm the conclusion of Hang et al. (1999) about a mid B-type underlying star in this source. Comparing V669 Cep with AS 381, in whose spectrum lines of the cool companion are seen redward of $\sim 5800 \AA$ and for which we estimated the companions' brightness ratio of $\Delta V \sim 2.2 \mathrm{mag}$, we can suggest that the brightness ratio in the V669 Cep system is even larger. In this case the cool companion should be a late-type subgiant or even a dwarf. Thus, taking the suggestion of Hang et al. (1999) that the hot companion is a pre-main-sequence Herbig Be star, it is natural to assume that the cool companion is a T Tau star.

The optical colour-indices (Table 1) and the bluest part of the near-IR spectrum (Fig. 3) indicate that the overall (inter- and circumstellar) reddening is rather low. At the same time, the steep rise of the observed flux from $\sim 1.5$ to $\sim 10 \mu \mathrm{m}$ is consistent with a large amount of dust in the system. It also indicates that the dust is most likely optically thick in the optical region. Two different explanations for this situation can be considered.

The first explanation concerns the presence of individual non-spherical envelopes around each companion or a circumbinary disc. Such a circumstellar matter distribution can be deduced from the double-peaked optical line profiles (Fig. 1). The disc (or discs) should be inclined to the line of sight at an intermediate angle, because the line profiles are narrow and the central depressions are shallow. We have already shown that the Brackett emission lines strength in the close to pole-on object AS 381 are much weaker than in the more equator-on MWC 657 (Miroshnichenko et al. 2002) both of which display properties, similar to those of V669 Cep. The line strength difference is mainly due to a stronger continuum, coming from a larger surface of a more inclined disc (if both the lines and continuum are formed in the same envelope). In V669 Cep the Brackett lines are a little stronger than those in AS 381 (see Fig. 4). Thus if the gas-and-dust envelope of V669 Cep is a disc viewed close to pole-on, it can obscure both companions a little. This does not contradict a possible pre-main-sequence nature of the system.

However, the $\mathrm{H} \alpha$ emission line is too strong for a mid B-type Herbig star, if we assume that the bulk of the circumstellar gas is illuminated by the hot companion alone (or the absence of the cool companion). Even more massive pre-main-sequence objects (such as HD 200775, Miroshnichenko et al. 1998, or MWC 297, Drew et al. 1997) rarely have a stronger $\mathrm{H} \alpha$ emission. If we attribute the line emission to the much fainter T Tau companion, this contradiction would become even stronger. The equivalent width of the $\mathrm{H} \alpha$ line would be $\geq 1000 \AA$, that is not observed in any of such objects (see Cohen \& Kuhi 1979). Other arguments against the pre-main-sequence nature of the object include the following:

1. both Herbig Ae/Be and T Tau stars usually have much flatter IR SED due to the presence of distant and cold circumstellar dust of protostellar origin. If the companions have the same age, the T Tau star, which evolves much slower than the intermediate-mass hot companion, has to be extremely young and exhibit a significant far-IR excess with a flux rising longward of $\sim 10 \mu \mathrm{m}$.

2. The CN (0-0) $1.1 \mu \mathrm{m}$ absorption band is a feature of luminous stars.

The second explanation assumes that the companions are obscured differently. Since we observe no significant obscuration of the hot companion (from the low optical reddening), the dust might obscure only the cool companion. The hot companion can even have no dust around it, because its relatively high $U V$ flux prevents the dust condensation in its Roche lobe. This interpretation is supported by the fact that we see only very weak [N II] nebular lines in the spectrum of V669 Cep (see Fig. 2b). Such a faint phenomenon implies that the circumstellar gas distribution around the hot star is rather compact, and its average density is relatively high. Therefore, the optical nebula around the object noticed by Hang et al. (1999) on the Palomar Sky Survey plate may represent the scattered light off the dust around the cool companion, which in this case can be intrinsically much brighter. It can be a giant as in the MWC 623 system (Zickgraf 2001). Since the dust condensation radius is very low for a cool star, all the dust in the system can be confined to the cool companion's Roche 
lobe. Such a model has been considered for some symbiotic systems (e.g., V1016 Cyg, Taranova \& Bogdanov 2001). This idea can be applied to other Be stars with warm dust and, hence, be used to correct the brightness ratios in known and suspected binaries. At the same time, we should note that these binaries cannot be fully identified with symbiotic systems. The former seem to have less evolved hot companions than the latter. Nevertheless, the evolutionary state of the Be stars with warm dust is not constrained yet and requires further investigation, which is out of this paper scope.

A suggestion that V669 Cep is an optical (projected) pair can easily be ruled out. None of the companions alone seem to take all the object's features without a contradiction, as we explained above. The probability of a positional coincidence of two stars, surrounded by a significant amount of circumstellar matter, in a relatively unpopulated field is extremely low.

The shape of the $\mathrm{H} \alpha$ profile (Fig. 2a) is suggestive of the presence of a broader double-peaked structure (at $\left.I / I_{\mathrm{c}} \sim 10\right)$ in addition to the strong and variable central peak. The double-peaked emission profile is a gaseous disc feature, while the central peak may be formed in a ring, close to the Roche lobe boundary of the hot companion and in a region of the mass transfer. The suggestion about mass transfer is justified by the presence of an extremely strong $\mathrm{H} \alpha$ emission, which seems to be too strong for a reasonable mass loss rate from a mid B-type star (as we mentioned above).

We can roughly estimate the parameters of the system and its stellar companions. The hot companion's luminosity $\log L / L_{\odot}=2.7 \pm 0.2$ can be derived from the above $M_{V}$, a B4-B 6 spectral type $\left(T_{\text {eff }} \sim 15000 \pm 2000 \mathrm{~K}\right.$, Straizys \& Kuriliene 1981), and a bolometric correction of $\sim-1$ mag (Miroshnichenko 1997). This leads to the star's radius $R_{*} \sim 3 R_{\odot}$ and mass $M_{*} \sim 4 M_{\odot}$. Then, if the companions have the same luminosities and masses (as in MWC 623, Zickgraf 2001), and the cool star has a $K$ spectral type (the CO bands are too faint for an M-type star, as in AS 381, Miroshnichenko et al. 2002), the minimum orbital period would be about 5 days (assuming that the stars touch each other). Since there is a large amount of circumstellar matter in the system, both companions need some space for its storage. This will make their separation and orbital period larger.

A very sparce light curve obtained by Takamizawa contains 2 brightenings of the object in early 1998 and mid 1999 ( $\sim 1.5$ years apart) by $0.3-0.4 \mathrm{mag}$. These are probably not related to the orbital rotation in the system, which does not seem to be eclipsing (see above arguments). However if the cool companion is more massive, has a larger Roche lobe, and the dust is distributed around it mostly spherically (as in the case of V1016 Cyg), then the dust can obscure the hot star at certain orbital phases even at relatively large inclinations $\left(\geq 30^{\circ}\right)$ of the orbital plane to the line of sight. The absence of detailed RV variation data does not allow us to give an accurate estimate for the orbital period. The existing $\mathrm{H} \alpha$ data (Table 5) show that significant intensity variations occur on a timescale of a few months. If mass loss or transfer is relatively stable in the system, these variations may be due to the projection or other effects, related to the orbital motion. From the fact that classical Be and Be/X-ray binaries with the strongest emission-line spectra have orbital periods of 150-250 days (Reig et al. 1997) and that the $\mathrm{H} \alpha$ line in V669 Cep is extremely strong, we can suggest that its oribtal period is of the order of a few hundred days. Such a period implies an angular separation of a few milliarcseconds at $D=1 \mathrm{kpc}$, that can be detected by the next generation satellites, such as SIM, or ground-based speckle interferometers (e.g., IOTA).

As we discussed in our previous papers (Miroshnichenko et al. 2000, 2002), the steep decrease of the far-IR flux probably implies that the system is still evolving redward from the main-sequence and is too young to produce a pronounced SED peak at $25-60 \mu \mathrm{m}$, which is observed in the IRAS data for post-AGB stars or LBVs. The presence of forbidden lines in the spectrum of V669 Cep allows us to classify it as a B[e] object, a new member of the group of Be stars with warm dust. We note here that despite our conclusion that the dust is most likely located around the cool companion, there is no contradiction with the $\mathrm{B}[\mathrm{e}]$ type definition (the presence of forbidden lines and dust, Allen \& Swings 1976), which does not specify the dust origin. In fact, the $\mathrm{B}[\mathrm{e}]$ star list contains a number of symbiotic stars, which are observationally similar to V669 Cep except for the reversed companions' brightness ratio.

\section{Conclusions}

We obtained and analysed optical and near-IR spectroscopic and photometric observations of the emission-line star V669 Cep $=3 \mathrm{~g} 71$ associated with the IR source IRAS $22248+6058$. The optical spectrum is dominated by weak permitted and forbidden Fe II emission lines in addition to the strong and double-peaked [O I] lines and extremely strong and variable $\mathrm{H} \alpha(66 \leq E W \leq 187 \AA)$. Both our optical photometry and spectrum suggest that a mid B-type (B4-B6) low-luminosity star, surrounded by a non-spherical gaseous envelope, is responsible for the observed features and SED in the optical domain. The absorption bands of $\mathrm{CN}$ and $\mathrm{CO}$, seen in the near-IR spectrum, as well as a steep rise of the observed flux in the 1.5-10 $\mu \mathrm{m}$ region indicate the presence of a cool (probably K-type) star in the system. The dusty envelope seems to be located mostly around the cool companion, reducing its brightness. The flux decrease longward of $10 \mu \mathrm{m}$ provides evidence that the dusty envelope is rather compact and may be confined within the Roche lobe of the cool companion. The $\mathrm{H} \alpha$ line strength suggests an ongoing mass exchange in the system, probably through the companions' circumstellar matter. The binary belongs to the local spiral arm at a distance of $1-1.5 \mathrm{kpc}$ from the Sun. 
The described properties of V669 Cep rule out the hypothesis by Hang et al. (1999) that it is a pre-mainsequence Herbig Be star. Instead, we argue that it is a post-main-sequence intermediate-mass binary, although probably less evolved than symbiotic systems. Further optical and IR observations of V669 Cep are very desirable in order to study its brightness and spectral variations in more detail.

Acknowledgements. We thank Jules Halpern for making his spectra of V669 Cep available to us and David Knauth for help with the McDonald data reduction. A. M. and K. S. B. acknowledge support from NASA grant NAG5-8054. Karen Bjorkman is a Cottrell Scholar of the Research Corporation, and gratefully acknowledges their support. This work was supported in part by the U.S. Civilian Recearch \& Development Foundation (CRDF) grant RP1-2264. P. G.-L. acknowledges support from grant PB97-1435-C02-02 from the Spanish Dirección General de Enseñanza Superior e Investigación Científica (DGESIC). R. J. R. and D. K. L. were supported by the Independent Research and Development program at The Aerospace Corporation. This research has made use of the SIMBAD database operated at CDS, Strasbourg, France.

\section{References}

Allen, D. A., \& Swings, J.-P. 1976, A\&A, 47, 293

Arribas, S., \& Martínez-Roger, C. 1987, A\&AS, 70, 303

Bessell, M. S., \& Brett, J. M. 1988, PASP, 100, 1134

Cohen, M., \& Kuhi, L. V. 1979, ApJS, 41, 743

Coluzzi, R. 1993, Bull. Inf. CDS, 43, 7

Drew, J. E., Bushfield, G., Hoare, M. G., et al. 1997, MNRAS, 286,538

Efremov, Yu. N. 1989, in Sites of Star Formation in Galaxies, Moscow (Nauka)

Egan, M. P., Price, S. D., Moshir, M. M., et al. 1999, The Midcourse Space Experiment Point Source Catalog Version 1.2, Explanatory Guide, AFRL-VS-TR-1999-1522

Gonzales, G., \& Gonzales, G. 1956, Bol. Obs. Tonantzintla y Tacubaya, No. 15, 16

Grandi, S. A. 1975, ApJ, 196, 465

Grandi, S. A. 1976, ApJ, 206, 658

Halpern, J., Gotthelf, E. V., Leighly, K. M., \& Hefland, D. J. 2001, ApJ, 547, 323

Hang, H.-R., Liu, Q.-Z., \& Xia, J.-P. 1999, A\&A, 344, 614

Herbig, G. H. 1993, ApJ, 407, 142

in't Zand, J. J. M., Halpern, J., Eracleous, M., et al. 2000, A\&A, 361, 85

IRAS Explanatory Supplement to the Catalogs and Atlasses 1985, ed. C. A. Beichman, G. Neugebauer, H. J. Habing, P. E. Clegg, \& T. J. Chester, NASA RP-1190

Ivezić, Ž., \& Elitzur, M. 1995, ApJ, 445, 415

Kazarovets, E. V., Samus, N. N., \& Durlevich, O. V. 2001, Inform. Bull. Var. Stars, No. 5135
Khaliullin, Kh., Mironov, A. V., \& Moshkaliov, V. G. 1985, Ap\&SS, 111, 291

Kornilov, V. G., Volkov, I. M., Zakharov, A. I., et al. 1991, Sternberg Astron. Inst. Proc., v. 63

Kurucz, R. L. 1994, Smithsonian Astrophys. Obs., CD-ROM No. 19

Lasker, B. M., Sturch, C. R., McLean, B. J., et al. 1990, AJ, 99, 2019

Lopes, D. F., Damineli Neto, A., \& Freitas Pacheko, J. A. 1992, A\&A, 261, 482

McCarthy, J. K., Sandiford, B. A., Boyd, D., \& Booth, J. 1993, PASP, 105, 881

Miroshnichenko, A. S. 1997, In Fundamental Stellar Parameters, ed. T. R. Bedding (Publ. School of Physics, Univ. of Sydney, Australia), Proc. IAU Symp., 189, 50

Miroshnichenko, A. S., Mulliss, C. L., Bjorkman, K. S., et al. 1998, PASP, 110, 883

Miroshnichenko, A. S., Chentsov, E. L., Klochkova, V. G., et al. 2000, A\&AS, 147, 5

Miroshnichenko, A. S., Levato, H., Bjorkman, K. S., \& Grosso, M. 2001, A\&A, 371, 600

Miroshnichenko, A. S., Bjorkman, K. S., Chentsov, E. L., et al. 2002, A\&A, 383, 171

Munch, G. 1957, ApJ, 125, 42

Panchuk, V. E., Najdenov, I. D., Klochkova, V. G., et al. 1998, Bull. Spec. Astrophys. Obs., 44, 127

Panchuk, V. E., Klochkova, V. G., Najdenov, I. D., et al. 1999, Preprint of the Special Astrophys. Observ., No. 139

Reig, P., Fabregat, J., \& Coe, M. J. 1997, A\&A, 322, 193

Rudy, R. J., Erwin, P., Rossano, G. S., \& Puetter, R. C. 1991, ApJ, 383, 344

Rudy, R. J., Mazuk, S., Puetter, R. C., \& Hamman, F. 2000, ApJ, 539, 166

Sheikina, T. A., Miroshnichenko, A. S., \& Corporon, P. 2000, in The Be-phenomenon in Early-Type Stars, ed. M. A. Smith, H. F. Henrichs, \& J. Fabregat, ASP Conf. Ser., 214, 494

Straizys, V. 1977, in Multicolour photometry of stars (Vilnius, Mokslas Publ.)

Straizys, V., \& Kuriliene, G. 1981, Ap\&SS, 80, 353

Taranova, O. G., \& Bogdanov, M. B. 2001, Astron. Rep., 45, 461

Tuohy, I. R., Buckley, D. A. H., Remillard, R. A., et al. 1988, in Physics of Neutron Stars and Black Holes, ed. Y. Tanaka (Universal Academy Press Inc., Tokyo), 93

Wackerling, L. R. 1970, Mem. RAS, 73, 153

Wallace, L., Meyer, M. R., Hinkle, K., \& Edwards, S. 2000, ApJ, 535, 325

Weaver, Wm. B., \& Jones, G. 1992, ApJS, 78, 239

Wegner, W. 1994, MNRAS, 270, 229

Wilson, J. C., Skrutskie, M. F., Colonno, M. R., et al. 2001, PASP, 113, 227

Wood, K. S., Meekins, J. F., Yentis, D. J., et al. 1984, ApJS, 56, 507

Zickgraf, F.-J. 2001, A\&A, 375, 122 